\title{
Les Intelligences célestes dans la poésie scientifique du xvième siècle
}

\author{
JAMES DAUPHINÉ
}

Les poètes scientifiques du XVI ${ }^{\text {ìme }}$ siècle, soucieux d'enquêter sur les mystères du cosmos et d'en déchiffrer les lois, n'ont ni limité leur investigation au domaine de la nature, ni adopté exclusivement dans leur recherche des principes rationnels et modernes. ${ }^{1}$ Pour eux, A.M. Schmidt l'a magistralement démontré, ${ }^{2}$ il importait par tous les moyens - de l'intuition à la foi, de l'imagination à la raison, de la tradition à l'inspiration originale - de parvenir à comprendre les mécanismes du monde comme à expliquer les beautés du microcosme. Guidés par Uranie qu'invoquait déjà Dante, ${ }^{3}$ ils ont eu à coeur de s'élever dans l'échelle de la connaissance, d'entrevoir les secrets divins et d'en proposer une analyse. C'est dans le cadre de cette démarche, plus intellectuelle que poétique, que nombre d'entre eux ont été amenés à méditer sur l'essence et la fonction des daimons et des anges auxquels on conférait le double pouvoir de gouverner, voire de mouvoir, un astre, une planète ou un ciel, et d'influencer le déroulement de la vie humaine. Le retour de ces thèmes, qu'illustre le motif des "intelligences célestes," toujours présent au sein des poèmes scientifiques, peut surprendre si l'on néglige les leçons du Timée et les commentaires néo-platoniciens qu'elles ont engendrés, si l'on méconnaît également l'importance que l'angéologie avait revêtu pour l'Eglise, du haut Moyen-Age à l'aube du XVII ${ }^{\text {ème }}$ siècle. ${ }^{4}$ S'interroger sur les daimons et les anges conduit à prendre en considération une vision cosmologique où la science des astres et celle de la musique rejoignent celle de l'Ecriture dans la démonstration, la louange ou tout simplement la reconnaissance de l'harmonie du monde.

L'influence du Timée et celle du célèbre traité attribué au pseudo-Denys l'Aréopagite, La Hiérarchie Céleste, ont singulièrement contribué à accroître dans le contexte catholique et romain le rôle des Intelligences régentes des planètes ou des cieux. Saint Thomas d'Aquin, parmi d'autres, avait accepté les conceptions de Denys et celles de Grégoire si répandues alors. ${ }^{5} \mathrm{Qu}$ 'on se rappelle les vers du Paradis où Dante s'est plu à exposer l'ordonnance des choeurs angéliques. ${ }^{6}$ Si le poète de La Divine Comédie, gravissant les cieux, 
était contraint de s'attacher à leur organisation, partie intégrante de sa vision et donc de sa transposition littéraire, il n'en allait pas de même des poètes scientifiques du XVI ${ }^{\text {ème }}$ siècle qui multiplièrent plutôt les rapprochements entre constellations, planètes et réalités spirituelles. Le statut du poète était autre: mais s'il n'était plus un voyant invité ou prédestiné à accomplir un voyage stellaire, il demeurait le secrétaire inspiré, animé par la furor qui lui permettait d'atteindre à une plus grande compréhension de l'univers. Ainsi, parce qu'il était enthousiasmé au sens étymologique de ce terme, le poète avait lui aussi charge de raconter ce qu'il avait vu, de fixer sur le papier le fonctionnement du grand monde, de faire oeuvre docte.

Particulièrement significatifs de ce projet sont ces vers de l'Hymne des Daimons, aujourd'hui trop souvent négligés, que Ronsard avait supprimés dans sa version finale:

Que les Anges mutins, qui ses compagnons furent,

Les uns en l'air, en l'eau, et sur la terre cheurent,

Et selon le forfaict de leurs commis pechez

Se veirent loin du Ciel, dans des corps attachez,

Qui servent de prisons à leur coulpe ancienne,

Jusques à-tant que Dieu juger le Monde vienne.

Ceux qui ont un corps d'air, ont la craincte de se voir

Prendre un terrestre corps, les terrestres de cheoir

Là-bas dans les Enfers, où le feu les consomme,

Quand pour punition ilz deçoivent un homme:

Car sans la peur qu'ilz ont, iamais ilz ne feroient

Que nous tenter l'esprit, et nous abuseroient.

D'aultres ont estimé qu'il n'y avoit Planette

Qui n'en eust dessouz elle une bande subjette,

Par qui sont les mortelz en vivant gouvernez

Selon l'Astre du ciel, soubz lequel ilz sont nez:

Ceux de Saturne font l'homme melancholique:

Ceux de Mars, bon guerrier, ceux de Venus, lubrique,

Ceux de la Lune, prompt: cauts les.Mercuriens,

Ceux du Soleil, aymé: heureux les Joviens:

L'un bon, l'autre est mauvais: le bon nous pousse à faire

Tout acte vertueux, le mauvais, au contraire. ${ }^{7}$

Ce passage, qui contient une liaison pour le moins malencontreuse et hâtive entre anges révoltés et daimons, s'il fut justement retiré d'un hymne consacré à ces derniers, atteste cependant la vitalité d'un raisonnement analogique cher au néo-platonisme ficinien ${ }^{8}$ et qui trouve écho un peu partout au XVI ${ }^{\text {ème }}$ siècle dans les oeuvres de Tyard, La Boderie, Vigenère ou Du Monin. Au XVII ${ }^{2}$ siècle encore, rien n'est plus naturel que de rencontrer sous la plume de théologiens ou de poètes la définition détaillée du lien unissant planètes et Intelligences, commme pourraient en témoigner surabondamment le célèbre traité de T. Heywood: The Hierarchie of the blessed Angells, their Names, Orders, and Offices. The Fall of Lucifer with his angells ${ }^{9}$ et les non moins 
fameuses tables du Père Kircher répertoriant le nom des anges, le siège et le champ planétaire de leur activité. ${ }^{10}$ Quant à l'ouvrage peu connu de J. Saulnier Cosmologie du Monde tant celeste que terrestre (...), il renfermait lui aussi, à l'instar de maints traités cosmologiques le rappel des rapports entre ciel et anges:

L'ordre que Dieu a mis aux susdits Cieux, leur donnant à chacun une intelligence ou Ange, comme au Ciel de Saturne Cassiel; à celuy de Iupiter Sachiel; à celui de Mars Samuel: à celuy du Soleil Michaël: à celuy de Venus Anahel: à celuy de Mercure Raphaël: et à celuy de la Lune Gabriel: le devoir desquels est, de les faire aller de telle vitesse, qu'il est presque incroyable de l'ouyr dire. ${ }^{11}$

Dans une perspective sensiblement identique on peut observer l'exploitation que fit Joseph Du Chesne de ce motif. Au Livre I de son encyclopédie Le Grand miroir du monde, conformément à la tradition hébraïque condamnée mais toujours vivante ${ }^{12}$ et qu'H. Corneille Agrippa avait reprise dans sa Philosophie Occulte, ${ }^{13}$ il écrivait:

Chasque estoile a le sien, douze ont leur domicile
Dans les douze maisons de l'Escharpe mobile,
Alchidaël se tient près de l'astré Belier,
Asmoriel secourt le Taureau printanier,
Ambriel les Bessons, Muriel l'Escrevice,
Vetchel le Lyon, Hamariel service
Fait à la Vierge Astree, et Zuriel tousiours
Esgale au Trebuschet les nuicts avec les iours,
A l'astre veneneux Barchiel obtempere,
Adnachiel les traits guide du Sagittaire,
Hamuel, Gambriel, Barchel sont gouverneurs
Du Chevreul, du Verseau, et des luisans Nageurs. ${ }^{14}$

Aucune originalité dans ces vers qui apparaissent régulièrement dans les oeuvres cosmologiques de la fin du XVI ${ }^{\text {eme }}$ siècle et qui n'apportent rien à la qualité poétique de l'inspiration et de son expression. Présents dans les corps célestes, daimons et anges étaient des garants de l'harmonie du monde: sans eux ou bien à cause de leur discorde les cieux s'entrechoquaient; avec eux et grâce à leur entente, se conservait l'harmonie de l'univers et se pérennisait le miracle de la création.

Lorsque les poètes scientifiques ont voulu définir ou illustrer la notion de cosmos, ils ont naturellement usé, parfois avec bien peu de discernement, d'allégories, métaphores et comparaisons empruntées au domaine musical: témoin l'image du luth de l'univers chère à La Boderie, Du Bartas, comme plus tard à Fludd ou au Père Mersenne. ${ }^{15}$ A la suite de George de Venise dont il avait traduit le de Harmonia mundi totius cantica tria (...), La Boderie, au fil de sa propre production, a abondamment utilisé raisonnements, analogies 
et images mettant en évidence la présence de lois harmoniques au sein de l'univers. Le champ sémantique de la musique et son usage systématique dans les oeuvres de La Boderie, Du Monin, Tyard, mais aussi de Benlowes et de T. Heywood, ${ }^{16}$ a abouti à d'interminables développements où il est difficile de suivre la progression de la pensée si l'on ne possède pas des notions précises de musique. Nul n'entre dans La Galliade, ou de la Révolution dans les arts et les sciences s'il n'est musicien!

Dans ce processus littéraire, la traversée de l'écriture a pour fonction de traduire les lois musicales qui régissent l'homme, le monde, les Intelligences ... Les anges et les choeurs angéliques reçoivent une note qui s'inscrit sur la gamme divine et sont parfois assimilés aux anges musiciens figurant sur les triptyques du Moyen-Age. Les daimons ne sont pas quant à eux admis à ce concert. Les recueils de poésie cosmologique de Du Monin, Tyard, La Boderie semblent toujours répéter le même message: les Intelligences chantent la gloire de Dieu.

Une telle vision cosmologique, à laquelle n'a participé que bien timidement le "nouvel Orphée," G.S. de Du Bartas, était prépondérante dans La Galliade de La Boderie et L'Uranologie de Du Monin. Le premier, au cours des Cercles de La Galliade, a multiplié poncifs musicaux rendant compte de l'harmonie du macrocosme et de celle du microcosme. Le second, également disciple d'une vision musicale de l'univers précisait:

\footnotetext{
Mais ces motets du Ciel ne sont pas composez

Par ces feus radieus d'ame et de sens cassés:

Ils ont pour entonner leurs musicalles bandes,

Maistres Musiciens, igenieus Orlandes,

Pedagogues de choeur, qui iouans à testons,

Aus Novices du Ciel font reprendre leurs tons:

Zaphchiel, precepteur du porte-faus ancêtre,

De son fiz Iupiter Zachariel le maitre,

Zamael gouverneur de Mavors: Michael

Le Regent de Titan, de Venus, Anael,

Raphael, instructeur du messager Mercure,

Et l'Ange Gabriel qui Phebe prend à cure.

Mais tous ces set regens ne font que regenter

Pour le divin ouir du Principal flater

Qui leur temple fournit de Messes, et Regales

De bouches et de vois à nulle vois esgalles.

C'est pour le seul AMOUR du Roi des ecussons

Qui planchent, radieus, les celestes maisons. ${ }^{17}$
}

La formulation contournée laisse cependant transparaître dans ces vers exemplaires une constante de l'imaginaire qui de Dante à La Boderie, de Du Monin au Père Le Moyne s'est maintenue: la reconnaissance de l'unité de la création par l'harmonie qui s'en dégage. Au-delà des écueils d'un style tantôt ampoulé, tantôt bas,on découvre ce désir de magnifier une vision de l'univers et de l'homme. 
Au coeur de la poésie scientifique, anges et daimons, par leur statut et leur fonction, perpétuent une antique tradition, une lointaine révélation: celle d'un monde où prime l'Intelligence. Qu'ils animent les constellations zodiacales, qu'ils résident dans les planètes ou qu'ils agissent sur le déroulement de l'histoire en fonction de cycles précis, notamment établis par Trithème, ${ }^{18}$ ils sont la preuve qu'à l'intérieur de toute la création existe une infinie correspondance, s'accomplit un projet, se manifeste la puissance du verbe. L'intérêt accordé aux Intelligences est donc l'expression d'une quête, dans le meilleur des cas; mais généralement il semblerait plutôt correspondre à une exigence du genre littéraire choisi: "poésie cosmologique." S'il est légitime de souligner l'utilisation systématique, et souvent fort banale, que les poètes scientifiques ont fait des Intelligences, il n'est pas indifférent de rappeler combien cela a pu favoriser le développement d'une écriture où les déterminations de la haute science, de l'imagination et de la foi devaient se fondre pour exalter le principe de la hiérarchie, source organisatrice de tout le cosmos. Connaître les Intelligences c'est déjà parcourir les visages du monde, en découvrir les lois et les secrets!

\section{UER des Lettres et Sciences Humaines de Nice}

\section{Notes}

1 T.S. Kuhn, The Copernican revolution (Cambridge: Havard University Press, 1957); C. Lewis, The Merton Tradition and Kinematics in Late Sixteenth Century Italy (Padoue: Antenore, 1980).

2 A.M. Schmidt, La Poésie scientifique en France au XVI ìme siècle (Paris: A. Michel, 1938).

3 Dante, La Divine Comédie, Purgatoire, XXIX, vers 40-42.

4 D.P. Walker, Spiritual and Demonic Magic from Ficino to Campanella (Londres: The Warburg Institute, vol. XXII, 1958).

5 J. Danielou, Les Anges et leur Mission d'après les Pères de l'Eglise (Paris: éd. de Chevetogne, 1951); G. Tavard, Les Anges (Paris: éd. du Cerf, 1971).

6 Dante, La Divine Comédie, Paradis, XXVIII, vers 98-135.

7 Ronsard, Oeuvres complètes, éd. Laumonier (Paris: Droz, 1935), tome 8, pp. 124-125, vers 179-200. Voir aussi H. Weber, La Création poétique au XVI ème siècle en France (...) (Paris: Nizet, 1956 [1955]), chap. VI, pp. 503-558: "La poésie cosmique et philosophique" et l'excellent article d'I. Silver, "Ronsard's Reflections on Cosmogony and Nature," PMLA, 79 (1965) 219-233.

8 M. Ficin, Théologie Platonicienne de l' immortalité des âmes (Paris: Les Belles Lettres, 1964), tome 1, Livre IV, chapitre I, pp. 152-153; D.P. Walker, op. cit. .

9 Londres: A. Islip, 1635.

10 A. Kircher, Oedipus aegyptiacus (...) (Rome, ex typ. V. Mascardi, 1652-654), tome 2, Class. IV Cabala Hebraeorum, cap. IX, p. 335; "Abacus Cabalicus Septenarij mystici." Class. V Cabala Saracenica, cap. VI, p. 385: Tabella II. continens septem Planetas cum literis, numeris, Angelis ipsis attributis, iuxta Arabum mentem, et Tabella III. continens 12 signa Zodiaci, una cum numeris, Literis Angelis, ex mente Arabum.

11 (Paris: Michel Daniel, 1618), VI-246 p., p. 28.

12 J. Dauphiné, "Sciences et Hiérarchie angélique," Actes du III ème Congrès néo-latin de Tours (sept. 1976) (Paris: Vrin, 1980), tome 2, pp. 831-848 où sont reproduites les planches du Septem principium angelorum orationes cum missa et eorum antiquis imaginibus d'A. del Duca, Rome, 1555.

13 H. Corneille Agrippa, La Philosophie Occulte (Paris: éd. Traditionnelles, 1962-1963), tome 3, Livre III, chap. XIV et XV. 


\section{6 / Renaissance and Reformation}

14 (Lyon: B. Honorat, 1587), Livre I, p.59.

15 J. Dauphiné, "L'image musicale de l'homme et du monde dans quelques textes du XVI ${ }^{\text {ème }}$ siècle," Annales de la Faculté des Lettres et Sciences Humaines de Nice, nº 22 (1974), pp. 53-68.

16 E. Benlowes, Sphinx Theologica, sive Musica Templi, ubi Discordia concors, in Tres Decades totidemque Libros divisa (Cambridge, 1636) Liber I. Supra note 9.

17 J.E. Du Monin, Le Quaresme (...) (Paris: J. Parent, 1584), p. 41.

$18 \mathrm{~J}$. Trithème, de Septem secunda deis id est intelligentiis sive spiritibus moventibus orbes libellus preciosissimus (Coloriae, apud J. Birckmannum, 1567 [1522]). 